\title{
Mean-Variance Portfolio Selection Problem with Stochastic Salary for a Defined Contribution Pension Scheme: A Stochastic Linear-Quadratic Framework
}

\author{
Charles I. Nkeki ${ }^{1,}$ * \\ ${ }^{1}$ Department of Mathematics, Faculty of Physical Sciences, University of Benin, Nigeria
}

Received 2 July 2013; Accepted 28 October 2013

Editor: Junfeng Yang

\begin{abstract}
This paper examines a mean-variance portfolio selection problem with stochastic salary and inflation protection strategy in the accumulation phase of a defined contribution (DC) pension plan. The utility function is assumed to be quadratic. It was assumed that the flow of contributions made by the pension plan members (PPMs) are invested into a market that is characterized by a cash account, an inflation-linked bond and a stock. In this paper, inflation-linked bond is traded and used to hedge inflation risks associated with the investment. The aim of this paper is to maximize the expected final wealth and minimize its variance. Efficient frontier for the three classes of assets that will enable PPMs to decide their own wealth and risk in their investment profile at retirement was obtained. The efficient frontier was found to be parabolic in shape, due to the present of initial capital and the existence of stochastic contributions of the PPM. Some numerical illustration of the analytical results are established in this paper.
\end{abstract}

Keywords mean-variance, stochastic salary, defined contribution, pension scheme, efficient frontier

DOI: $10.19139 /$ soic.v1i1.20

\section{Introduction}

In this paper, we consider a mean-variance portfolio optimization method with stochastic salary for a DC pension plan. This method is based on the pioneering

\footnotetext{
${ }^{*}$ Correspondence to: Department of Mathematics, Faculty of Physical Sciences, University of Benin, P. M. B. 1154, Benin City, Edo State, Nigeria Email: nkekicharles2003@yahoo.com
}

ISSN 2310-5070 Copyright (c) 2013 International Academic Press 
work of [18] and [19], the Nobel pricing-winning economist, widely recognized as the father of modern portfolio theory.

Today, the world is shifting from the international Pay-As-You-Go public pension scheme to DC pension scheme as a result of the evolution of the equity market. For example, in June 25, 2004, Nigeria replaced her government operation of pension scheme (i.e., Pay-As-You-Go pension scheme) with a privately managed system through making compulsory contribution into their retirement savings account (RSA). This scheme was established by the Nigerian Pension Reform Act (NPRA), 2004,[25]. The aims and objectives of the NPRA are: to ensure that every person who worked in either the public service of the Federation, Federal Capital Territory or private sector receives his/her retirement benefits as and when due; to assist improvident individual by ensuring that they save in order to cater for their livelihood during old age; and to establish a uniform set of rules, regulations and standards for the administration and payments of retirement benefits for the public service of the Federation, Federal Capital Territory and the private sectors. The PPMs make a continuous stochastic income stream into the pension scheme. This cash inflows of which the PPM bears a considerable risk on can be affected by inflation risk. Hence, the need to hedge such risk has become imperative. In this paper, we introduced inflation-linked financial derivatives as bonds in order to hedge the inflation risk to investment

There are extensive literature that exist on the area of accumulation phase of a DC pension plan and optimal investment strategies which are given briefly highlighted below. For instance, [6] studied optimal dynamic asset allocation problem for defined contribution pension plans. [8] considered dynamic asset allocation problem in a mean-variance framework. [4] considered optimal funding and investment portfolio strategies in defined contribution pension plans under Epstein-Zin utility. [2] studied optimal pension fund management strategies in a stochastic framework. [5] considered optimal management under stochastic interest rates in a defined contribution pension fund in which the plan member is protected from interest rate risk. [10] examined pension funds with a minimum guarantee under a stochastic control approach. [13] considered optimal investment strategies and risk measures in defined contribution pension schemes. [23] considered the efficiency of mean-variance based portfolio selection in DC pension schemes. [11] studied stochastic optimal control of DC pension funds. [9] considered stochastic optimal control approach involving annuity contracts. [1] considered dynamic asset allocation in a mean-variance framework. [20] considered optimal portfolio management of the accumulation phase of a defined contributory pension scheme. [21] studied a variational form of classical portfolio strategy and expected wealth for a defined contributory pension scheme.

In the context of DC pension plans, the problem of finding the optimal investment strategy with stochastic salary and inflation protection under meanvariance efficient approach has not been reported in published articles. [14] and 
[23] assumed a constant flow of contributions into the pension scheme which will not be applicable to salary earners in pension scheme. We assume that the contribution of the PPM grows as the salary grows over time.

In the literature, the problem of determining the minimum variance on trading strategy in continuous-time framework has been studied by [22] via the martingale approach. [1] used the same approach in a more general framework. [17] solved a mean-variance optimization problem in a discrete-time multi-period framework. [24] considered a mean-variance in a continuous-time framework. They show the possibility of transforming the difficult problem of mean-variance optimization problem into a tractable one, by embedding the original problem into a stochastic linear-quadratic control problem, that can be solved using standard methods. These approaches have been extended and used by many in the financial literature, see for instance, [23], [3], [15], [14] and [7]. In this paper, we study a meanvariance approach (MVA) to portfolio selection problem with stochastic salary of a PPM and inflation protection strategy in accumulation phase of a DC pension scheme. Our result shows that inflation-linked bond can be used to hedge inflation risk that is associated with the PPM's wealth. We found that our optimal portfolio is efficient in the mean-variance approach.

The remainder of this paper is organized as follows. In section 2, we present the problem formulation and financial market models. We also establish in this section, the dynamics of PPM's stochastic salary and the wealth process of a PPM. In section 3, we present the mean-variance approach of our problem. In section 4 , we present the optimization processes of our problem and expected utility of PPM's wealth at time $t$. Also in this section, we present the portfoilo process of the PPM at time $t$. In section 5, we present the expected terminal wealth for the PPM. In section 6, we present the efficient frontier of the PPM's wealth at terminal period. Section 7 presents the numerical illustration of our results. Finally, section 8 concludes the paper.

\section{Problem Formulation}

Let $(\Omega, \mathcal{F}, \mathbf{P})$ be a probability space. In this paper, the sign ' denotes transpose. Let $\mathbf{F}(\mathcal{F})=\left\{\mathcal{F}_{t}: t \in[0, T]\right\}$, where $\mathcal{F}_{t}=\sigma(W(s): s \leq t)$, where the Brownian motion $W(t)=\left(W^{B}(t), W^{S}(t)\right)^{\prime}, 0 \leq t \leq T$ is a 2-dimensional process, defined on a given filtered probability space $(\Omega, \mathcal{F}, \mathbf{F}(\mathcal{F}), \mathbf{P})$, where $\mathbf{P}$ is the real world probability measure and $\sigma(t)$ and $\sigma_{I}(t)$ are the volatility vectors of stock and volatility of the inflation-linked bond with respect to changes in $W^{S}(t)$ and $W^{B}(t)$, respectively, referred to as the coefficients of the market and are progressively measurable with respect to the filtration $\mathcal{F}$. 


\subsection{The financial models}

In this paper, we assume that the pension fund manager faces a market that is characterized by a risk-free asset (cash account) and two risky assets, all of whom are trade-able. We allow the stock price to be correlated to inflation. Also, we correlate the salary process of the PPM to stock. Therefore, the dynamics of the underlying assets are given in (1) to (3)

$$
\begin{gathered}
d B(t)=r(t) B(t) d t, B(0)=1, \\
d S(t)=S(t)(\mu(t) d t+\sigma(t) d W(t)), S(0)=s_{0} \in \mathbb{R}_{+}, \\
d Z(t, I(t))=Z(t, I(t))\left(\left(r(t)+\sigma_{B}(t) \theta_{B}(t)\right) d t+\sigma_{I}(t) d W(t)\right), \\
Z(0, I(0))=z_{0} \in \mathbb{R}_{+},
\end{gathered}
$$

where, $S(t)$ is stock price process at time $t, Z(t, I(t))$ is the price of inflationlinked bond, where $I(t)$ inflation index at time $t, \mu(t)$ is the appreciation rate for stock, $\sigma(t)=\left(\rho \sigma_{S}(t), \sqrt{1-\rho^{2}} \sigma_{S}(t)\right), \sigma_{I}(t)=\left(\sigma_{B}(t), 0\right), 0<\rho<1, r(t)$ is the nominal interest rate, $\theta_{B}(t)$ is the price of inflation risk, $B(t)$ is the price process of the cash account at time $t, S(t)$ is stock price process at time $t, I(t)$ is the inflation index at time $t$ and has the dynamics:

$$
d I(t)=E(\hat{r}(t)) I(t) d t+\sigma_{B}(t) I(t) d W^{B}(t),
$$

where $E(\hat{r}(t))$ is the expected rate of inflation, which is the difference between nominal interest rate, $r(t)$ and real interest rate $\tilde{r}(t)$ (i.e. $E(\hat{r}(t))=r(t)-\tilde{r}(t))$. $Z(t, I(t))$ is the inflation-indexed bond price process at time $t$.

Then, the volatility matrix

$$
\Sigma(t):=\left(\begin{array}{cc}
\sigma_{B}(t) & 0 \\
\rho \sigma_{S}(t) & \sqrt{1-\rho^{2}} \sigma_{S}(t)
\end{array}\right)
$$

corresponding to the two risky assets and satisfies $\operatorname{det}(\Sigma(t))=$ $\sigma_{S}(t) \sigma_{B}(t) \sqrt{1-\rho^{2}} \neq 0$. Therefore, the market is complete and there exists a unique market price $\theta(t)$ satisfying

$$
\theta(t):=\left(\begin{array}{c}
\theta_{B}(t) \\
\theta_{S}(t)
\end{array}\right)=\left(\begin{array}{c}
\theta_{B}(t) \\
\frac{\mu(t)-r(t)-\theta_{B}(t) \rho \sigma_{S}(t)}{\sigma_{S}(t) \sqrt{\left(1-\rho^{2}\right)}}
\end{array}\right)
$$

where $\theta_{S}(t)$ is the market price of risks.

\subsection{The stochastic salary of a PPM}

We assume in this paper that the salary process $Y(t)$ at time $t$ of the PPM is governed the by the dynamics

$$
d Y(t)=Y(t)\left(\beta(t) d t+\sigma_{Y}(t) d W(t)\right), Y(0)=y_{0} \in \mathbb{R}_{+},
$$


where $\beta(t) \in \mathbb{R}_{+}$is the expected growth rate of the salary of the PPM and $\sigma_{Y}(t)=\left(\sigma_{Y_{1}}(t), \sigma_{Y_{2}}(t)\right)$ is the volatility of the buying power of a PPM's salary. $\sigma_{Y_{1}}(t)$ is the volatility caused by the source of inflation, $W^{B}(t)$ and $\sigma_{Y_{2}}(t)$ is the volatility caused by the source of uncertainty arising from the stock market, $W^{S}(t)$. In this paper, we assume that $\sigma(t), \sigma_{I}(t), \sigma_{B}(t), \sigma_{S}(t), \sigma_{Y}(t), \mu(t), r(t)$, $\theta_{B}(t), \theta_{S}(t), \beta(t), \tilde{r}(t)$ are constants in time.

\section{Remark 1}

If the salary of a PPM is deterministic, then (6) becomes

$$
d Y(t)=\beta(t) Y(t) d t, Y(0)=y_{0} \in \mathbb{R}_{+} .
$$

Applying Itô Lemma on (6), we have the following

$$
Y(t)=y_{0} \exp \left(\left(\beta-\frac{1}{2}\left\|\sigma_{Y}\right\|^{2}\right) t+\sigma_{Y} W(t)\right) .
$$

\subsection{The wealth process}

Let $c \in \mathbb{R}_{+}$be the proportion of the PPM salary that is contributed into the pension plan, then the amount of contributions made by the PPM is $c Y(t)$ at time $t$. Let $X(t)$ be the wealth process of the PPM at time $t$ and $\Delta(t)=\left(\Delta^{B}(t), \Delta^{S}(t)\right)$ be the portfolio process at time $t$ such that $\Delta^{B}(t)$ is the fund invested in the inflationlinked bond at time $t$ and $\Delta^{S}(t)$, the fund invested in stock at time $t$. Then, $\Delta_{0}(t)=X(t)-\Delta^{B}(t)-\Delta^{S}(t)$ is the fund invested in cash account at time $t$. Therefore, the wealth process of the PPM is governs by the stochastic differential equation (SDE):

$$
\begin{aligned}
& d X(t)=(r X(t)+\Delta(t) \lambda) d t+\left(\Sigma^{\prime} \Delta^{\prime}(t)\right)^{\prime} d W(t), \\
& X(0)=x_{0} \in \mathbb{R}_{+},
\end{aligned}
$$

where, $\lambda=\left(\sigma_{B} \theta_{B}, \mu-r\right)^{\prime}$. Let $\bar{X}(t)=X(t)+c Y(t)$ be the total wealth of the PPM. Then, the total wealth dynamics of the PPM is given by

$$
\begin{aligned}
& d \bar{X}(t)=(r \bar{X}(t)+\Delta(t) \lambda+c(\beta-r) Y(t)) d t+\left(c \sigma_{Y}^{\prime} Y(t)+\Sigma^{\prime} \Delta^{\prime}(t)\right)^{\prime} d W(t), \\
& \bar{X}(0)=\bar{x}_{0}=x_{0}+c y_{0} \in \mathbb{R}_{+},
\end{aligned}
$$

The amount $\bar{x}_{0}$ is the initial fund paid into PPM's account. If no amount is paid into the PPM account at the beginning, then the initial wealth is null (i.e., $\bar{x}_{0}$ ). But, in this paper, we assume that at the beginning of the planning horizon, $\bar{x}_{0}$ amount of money is paid into the PPM's account.

\section{The Mean-Variance Approach}

In this section, we assume that the PPM invests his/her contributions through the PFA from time 0 to time $T$. The aim of the PPM is to maximize his/her expected 
terminal wealth and simultaneously minimize the variance of the terminal wealth. Hence, the PPM aim at minimizing the vector

$$
[-E(\bar{X}(T)), \operatorname{Var}(\bar{X}(T))]
$$

Definition 1: The portfolio strategy $\Delta()=.\left(\Delta^{B}(.), \Delta^{S}(.)\right)^{\prime}$ is said to be admissible if $\Delta(.) \in L_{\mathcal{F}}^{2}(0, T ; \mathbb{R})$ such that $\Delta^{B}(.) \in L_{\mathcal{F}}^{2}(0, T ; \mathbb{R})$ and $\Delta^{S}(.) \in$ $L_{\mathcal{F}}^{2}(0, T ; \mathbb{R})$.

Definition 2: The mean-variance optimization problem is defined as

$$
\min _{\Delta \in\left(\Delta^{B}, \Delta^{S}\right)} J=[-E(\bar{X}(T, \Delta)), \operatorname{Var}(\bar{X}(T, \Delta))]
$$

subject to:

$$
\left\{\begin{array}{cc}
\Delta(.), & \text { set of admissible portfolio strategy } \\
\bar{X}(.), \Delta(.), & \text { satisfy }(10) .
\end{array}\right.
$$

Remark: A portfolio is said to be efficient if it is not possible to have a higher return without increasing standard deviation.

Solving (11) is equivalent to solving the following equation

$$
\min _{\Delta \in\left(\Delta^{B}, \Delta^{S}\right)} J=[-E(\bar{X}(T, \Delta(.)))+\psi \operatorname{Var}(\bar{X}(T, \Delta(.)))], \psi>0,
$$

see [24].

By definition of variance in elementary statistics, we have

$$
\operatorname{Var}(\bar{X}(T))=E\left(\bar{X}(T)^{2}\right)-(E(\bar{X}(T)))^{2}
$$

Substituting (13) into (12), we obtain

$$
\min _{\Delta(.)} J=E\left[\psi \bar{X}^{2}(T)-\phi \bar{X}(T)\right]
$$

where,

$$
\phi=1+2 \psi E(\bar{X}(T)) .
$$

(14) is known as a linear-quadratic control problem. Hence, instead of solving (12), we now solve the following

$$
\min (J(\Delta(.)), \psi, \phi)=E\left[\psi \bar{X}(T, \Delta(.))^{2}-\phi \bar{X}(T, \Delta(.))\right]
$$

subject to:

$$
\left\{\begin{array}{cc}
\Delta(.), & \text { set of admissible portfolio strategy } \\
\bar{X}(.), \Delta(.), & \text { satisfy }(10) .
\end{array}\right.
$$




\section{The Optimization Problem}

In solving (16), we set $\omega=\frac{\phi}{2 \psi}$ and $F(t)=\bar{X}(t)-\omega$, see [14], [23] and [24].

Therefore, our problem is equivalent to solving

$$
\min _{\Delta(.)} J(\Delta(.), \psi, \phi)=\left[\frac{\psi F(T)^{2}}{2}\right],
$$

see [24] for details, where the process $F(t)$, which is the total wealth under the standard optimal stochastic control problem is given by the SDE:

$$
\left\{\begin{array}{l}
d F(t)=(r(F(t)+\omega)+\Delta(t) \lambda)+c(\beta-r) Y(t)) d t \\
\left.+\left(c \sigma_{Y}^{\prime} Y(t)+\Sigma^{\prime} \Delta^{\prime}(t)\right)\right)^{\prime} d W(t) \\
F(0)=f_{0}
\end{array}\right.
$$

Let

$$
U(t, f, y)=\inf _{\Delta(.)} E_{t, f, y}\left[\frac{\psi F(T)^{2}}{2}\right]=\inf _{\Delta(.)} J(\Delta(.), \psi, y)
$$

Then, the value function $U$ satisfies the following Hamilton-Jacobi-Bellman (HJB) equation:

$$
\left\{\begin{array}{l}
\inf _{\Delta \in \mathbb{R}}\left\{U_{t}+r(f+\omega) U_{f}+\Delta(t) \lambda U_{f}+c \beta y U_{y}-r c y U_{f}\right. \\
+\frac{1}{2} c^{2} y^{2} \sigma_{Y} \sigma_{Y}^{\prime} U_{y y}+c y \sigma_{Y}\left(\Sigma^{\prime} \Delta^{\prime}(t)\right) U_{f y} \\
\left.+\frac{1}{2}\left(\Sigma^{\prime} \Delta^{\prime}(t)\right)^{\prime}\left(\Sigma^{\prime} \Delta^{\prime}(t)\right) U_{f f}\right\}=0, \\
U(T, f, y)=\frac{1}{2} \psi f^{2} .
\end{array}\right.
$$

Assuming $U$ to be a convex function of $f$, then first order conditions lead to the optimal fraction of portfolio vector to be invested in inflation-linked bond and stock at time $t$ :

$$
\Delta^{\prime *}(t)=\frac{-\left(\Sigma \Sigma^{\prime}\right)^{-1}\left(\lambda U_{f}+c y \Sigma \sigma_{Y}^{\prime} U_{f y}\right)}{U_{f f}} .
$$

Substituting (21) into (20), we have

$$
\begin{aligned}
& U_{t}+r(f+\omega) U_{f}+c \beta y U_{y}-c r y U_{f}-\frac{(M \lambda)^{\prime} \lambda U_{f}^{2}}{2 U_{f f}} \\
& -\frac{c y\left(M \Sigma \sigma_{Y}^{\prime}\right)^{\prime} \lambda U_{f} U_{f y}}{U_{f f}}-\frac{c^{2} y^{2} \sigma_{Y} \sigma_{Y}^{\prime} U_{f y}^{2}}{2 U_{f f}}+\frac{1}{2} c^{2} y^{2} \sigma_{Y} \sigma_{Y}^{\prime} U_{y y}=0,
\end{aligned}
$$

where, $M=\left(\Sigma \Sigma^{\prime}\right)^{-1}$.

In this paper, we assume a quadratic utility function of the form:

$$
U(t, f, y)=P(t) f^{2}+Q(t) f y+u(t) y^{2}+(R(t) f+v(t) y)+C(t) .
$$


Finding the partial derivative of $U$ in (23) with respect to $t, f, f f, f y, y$, and $y y$, substitute into (22) and then extract the coefficients, we have the following system of ordinary differential equations (ODEs):

$$
\begin{gathered}
\frac{d P(t)}{d t}+a P(t)=0, P(T)=\frac{1}{2} \psi ; \\
\frac{d Q(t)}{d t}+k Q(t)-2 c r P(t)=0, Q(T)=0 ; \\
\frac{d R(t)}{d t}+r R(t)+2 r \omega P(t)-(M \lambda)^{\prime} \lambda R(t)=0, R(T)=0 ; \\
\frac{d v(t)}{d t}+r \omega Q(t)+c \beta v(t)-c r R(t)-\frac{(M \lambda)^{\prime} \lambda Q(t) R(t)}{2 P(t)} \\
-\frac{c\left(M \Sigma \sigma_{Y}^{\prime}\right)^{\prime} \lambda Q(t) R(t)}{2 P(t)}=0, v(T)=0 ; \\
\frac{d u(t)}{d t}+2 c \beta u(t)-c r Q(t)-\frac{(M \lambda)^{\prime} \lambda Q(t)^{2}}{4 P(t)}-\frac{c\left(M \Sigma \sigma_{Y}^{\prime}\right)^{\prime} \lambda Q(t)^{2}}{2 P(t)} \\
-\frac{c^{2} \sigma_{Y} \sigma_{Y}^{\prime} Q(t)^{2}}{4 P(t)}+c^{2} \sigma_{Y} \sigma_{Y}^{\prime} u(t)=0, u(T)=0 ; \\
\frac{d C(t)}{d t}+r \omega R(t)-\frac{(M \lambda)^{\prime} \lambda R(t)^{2}}{4 P(t)}=0, C(T)=0,
\end{gathered}
$$

where $k=r+c \beta-(M \lambda)^{\prime} \lambda-c\left(M \Sigma \sigma_{Y}^{\prime}\right)^{\prime} \lambda, \quad a=2 r-(M \lambda)^{\prime} \lambda$. Solving the systems of ODEs in (24) to (26), we have the following:

$$
\begin{gathered}
P(t)=\frac{1}{2} \psi e^{a(T-t)}, \\
Q(t)=\frac{c r \psi\left[e^{(k-a) T}-e^{(k-a) t}\right] e^{k t+a T}}{a-k}, \\
R(t)=\psi \omega\left(e^{r T}-e^{r t}\right) e^{a(T-t)-r T} \\
v(t)=\int_{t}^{T} e^{c \beta(\tau-t)}\left(c r R(\tau)-r \omega Q(\tau)+(r+c \beta-k) \frac{Q(\tau) R(\tau)}{2 P(\tau)}\right) d \tau . \\
u(t)=\int_{t}^{T} e^{\left(2 c \beta+c^{2} \sigma_{Y} \sigma_{Y}^{\prime}\right)(\tau-t)}(c r Q(\tau)+ \\
\left.\left((M \lambda)^{\prime} \lambda+2 c\left(M \Sigma \sigma_{Y}^{\prime}\right)^{\prime} \lambda+c^{2} \sigma_{Y} \sigma_{Y}^{\prime}\right) \frac{Q(\tau)^{2}}{4 P(\tau)}\right) d \tau \\
C(t)=\int_{t}^{T}\left(r \omega R(\tau)-\frac{(M \lambda)^{\prime} \lambda R(\tau)^{2}}{4 P(\tau)}\right) d \tau
\end{gathered}
$$




\subsection{Expected utility of wealth and optimal portfolio}

Therefore, the expected value of the utility of wealth of the PPM is

$$
\begin{aligned}
& U(t, f, y)=\frac{1}{2} f^{2} \psi e^{a(T-t)}+\frac{f y c r \psi\left[e^{(k-a) T}-e^{(k-a) t}\right] e^{k t+a T}}{a-k}+ \\
& y^{2} \int_{t}^{T} e^{\left(2 c \beta+c^{2} \sigma_{Y} \sigma_{Y}^{\prime}\right)(\tau-t)}\left(\operatorname{cr} Q(\tau)+\left((M \lambda)^{\prime} \lambda+2 c\left(M \Sigma \sigma_{Y}^{\prime}\right)^{\prime} \lambda+c^{2} \sigma_{Y} \sigma_{Y}^{\prime}\right) \frac{Q(\tau)^{2}}{4 P(\tau)}\right) d \tau \\
& +y \int_{t}^{T} e^{c \beta(\tau-t)}\left(\operatorname{cr} R(\tau)-r \omega Q(\tau)+(r+c \beta-k) \frac{Q(\tau) R(\tau)}{2 P(\tau)}\right) d \tau \\
& +f \psi \omega\left(e^{r T}-e^{r t}\right) e^{a(T-t)-r T}+\int_{t}^{T}\left(r \omega R(\tau)-\frac{(M \lambda)^{\prime} \lambda R(\tau)^{2}}{4 P(\tau)}\right) d \tau .
\end{aligned}
$$

At time $t=0,(36)$ becomes

$$
\begin{aligned}
& U\left(0, f_{0}, y_{0}\right)=\frac{1}{2} f_{0}^{2} \psi e^{a T}+\frac{f_{0} y_{0} c r \psi\left[e^{(k-a) T}-1\right] e^{a T}}{a-k}+ \\
& y_{0}^{2} \int_{0}^{T} e^{\left(2 c \beta+c^{2} \sigma_{Y} \sigma_{Y}^{\prime}\right) \tau}\left(\operatorname{cr} Q(\tau)+\left((M \lambda)^{\prime} \lambda+2 c\left(M \Sigma \sigma_{Y}^{\prime}\right)^{\prime} \lambda+c^{2} \sigma_{Y} \sigma_{Y}^{\prime}\right) \frac{Q(\tau)^{2}}{4 P(\tau)}\right) d \tau \\
& +y_{0} \int_{0}^{T} e^{c \beta \tau}\left(\operatorname{cr} R(\tau)-r \omega Q(\tau)+(r+c \beta-k) \frac{Q(\tau) R(\tau)}{2 P(\tau)}\right) d \tau \\
& +f_{0} \psi \omega\left(e^{r T}-1\right) e^{a T-r T}+\int_{0}^{T}\left(r \omega R(\tau)-\frac{(M \lambda)^{\prime} \lambda R(\tau)^{2}}{4 P(\tau)}\right) d \tau
\end{aligned}
$$

We observe that our utility function $U$ is indeed convex, since

$$
U_{f f}=2 P(t)>0, U_{f y}=Q(t)>0, U_{f f}=2 u(t)>0 .
$$

Now, substituting partial derivative of $U$ into (21), we have the following optimal portfolio of a PPM to be:

$$
\Delta^{\prime *}(t)=-M \lambda\left((f+\omega)-\omega e^{-r(T-t)}+c y g(t)\right)-c^{2} y M \Sigma \sigma_{Y}^{\prime} g(t),
$$

where $g(t)=\frac{r\left[e^{(k-a) T}-e^{(k-a) t}\right] e^{(k+a) t}}{a-k}$.

Hence, substituting $\bar{X}^{*}(t)$ for $f+\omega$ and $Y(t)$ for $y$ in (39), we have the following:

$$
\Delta^{\prime *}(t)=-M \lambda\left(\left(\bar{X}^{*}(t)-\omega e^{-r(T-t)}+c Y(t) g(t)\right)-c^{2} Y(t) M \Sigma \sigma_{Y}^{\prime} g(t) .\right.
$$

At $t=0$, we have

$$
\Delta^{\prime *}(0)=-M \lambda\left(\left(\bar{x}_{0}-\omega e^{-r T}+c y_{0} g(0)\right)-c^{2} y_{0} M \Sigma \sigma_{Y}^{\prime} g(0) .\right.
$$




\section{Expected Terminal Wealth for the PPM}

In this section, we consider the expected wealth and the second moment of the expected wealth for the PPM at time $t$. The evolution of the optimal stochastic wealth $\bar{X}^{*}(t)$ for a PPM under optimal feedback control $\Delta^{\prime *}(t)$ can be obtained by substituting (40) into (10) to obtain:

$$
\begin{aligned}
& d \bar{X}^{*}(t)= \\
& {\left[(a-r) \bar{X}^{*}(t)+(M \lambda)^{\prime} \lambda \omega e^{-r(T-t)}+c Y(t)(\beta-r-(r+c \beta-k) g(t))\right] d t} \\
& +\left[\Sigma^{\prime} M \lambda\left(\omega e^{-r(T-t)}-\bar{X}^{*}(t)\right)+c Y(t)\left(\sigma_{Y}^{\prime}-\gamma g(t)\right)\right]^{\prime} d W(t),
\end{aligned}
$$

where, $\gamma=\Sigma^{\prime} M \lambda+c \sigma_{Y}^{\prime}$. By applying Itô Lemma on (42), we obtain the SDE that governs the evolution of the square of optimal control $\bar{X}^{*}(t)$ :

$$
\begin{aligned}
& d \bar{X}^{* 2}(t)=\left[a \bar{X}^{* 2}(t)+c(\beta-r-(r+c \beta-k) g(t)\right. \\
& \left.-2 c\left(\Sigma^{\prime} M \lambda\right)\left(\sigma_{Y}^{\prime}-\gamma g(t)\right)\right) Y(t) \bar{X}^{*}(t)+2 c Y(t)\left(\Sigma^{\prime} M \lambda\right)^{\prime}\left(\sigma_{Y}^{\prime}-\gamma g(t)\right) \omega e^{-r(T-t)} \\
& \left.+(M \lambda)^{\prime} \lambda \omega^{2} e^{-2 r(T-t)}+G(t)\right] d t+2 \bar{X}^{*}(t)\left[\Sigma^{\prime} M \lambda\left(\omega e^{-r(T-t)}-\bar{X}^{*}(t)\right)\right. \\
& \left.+c Y(t)\left(\sigma_{Y}^{\prime}-\gamma g(t)\right)\right]^{\prime} d W(t)
\end{aligned}
$$

where $G(t)=c^{2} Y^{2}(t)\left(\sigma_{Y}^{\prime}-\gamma g(t)\right)^{\prime}\left(\sigma_{Y}^{\prime}-\gamma g(t)\right)$.

(43) is composed of the product of the optimal stochastic wealth, $\bar{X}^{*}(t)$ and the stochastic salary, $Y(t)$. Applying Itô to the product, we have

$$
\begin{aligned}
& d\left(Y(t) \bar{X}^{*}(t)\right)=\left[\left(a-r+\beta-\sigma_{Y}\left(\Sigma^{\prime} M \lambda\right)\right) Y(t) \bar{X}^{*}(t)\right. \\
& \left.+Y(t) \omega \sigma_{Y}\left(\Sigma^{\prime} M \lambda\right) e^{-r(T-t)}+c Y^{2}(t) \sigma_{Y}\left(\sigma_{Y}^{\prime}-\gamma g(t)\right)\right] d t \\
& +\left[\Sigma^{\prime} M \lambda\left(\omega Y(t) e^{-r(T-t)}-Y(t) \bar{X}^{*}(t)\right)+c Y^{2}(t)\left(\sigma_{Y}^{\prime}-\gamma g(t)\right)\right]^{\prime} d W(t)
\end{aligned}
$$

Taking the expectation on both sides of (42), (43), and (44), we find that the expected value of the optimal wealth, expected value of product of the stochastic wealth and the salary process, and the expected value of the square of optimal wealth and are given by the following ODEs:

$$
\begin{aligned}
& \left\{\begin{array}{l}
d E\left(\bar{X}^{*}(t)\right)=E\left[(a-r) \bar{X}^{*}(t)+(M \lambda)^{\prime} \lambda \omega e^{-r(T-t)}+c \eta(t) Y(t)\right] d t, \\
E\left(\bar{X}^{*}(0)\right)=\bar{x}_{0} \in \mathbb{R}_{+} \cdot
\end{array}\right. \\
& \left\{\begin{array}{l}
d E\left(Y(t) \bar{X}^{*}(t)\right)=E\left[\xi Y(t) \bar{X}^{*}(t)+Y(t) \omega \sigma_{Y}\left(\Sigma^{\prime} M \lambda\right) e^{-r(T-t)}\right. \\
\left.+c Y^{2}(t) \sigma_{Y}\left(\sigma_{Y}^{\prime}-\gamma g(t)\right)\right] d t, \\
E\left(Y(t) \bar{X}^{*}(0)\right)=\bar{x}_{0} y_{0} \in \mathbb{R}_{+} \cdot
\end{array}\right. \\
& \left\{\begin{array}{l}
d E\left(\bar{X}^{* 2}(t)\right)=E\left[a \bar{X}^{* 2}(t)+c \eta(t) Y(t) \bar{X}^{*}(t)+2 c Y(t) \pi(t) \omega e^{-r(T-t)}\right. \\
\left.+(M \lambda)^{\prime} \lambda \omega^{2} e^{-2 r(T-t)}+G(t)\right] d t, \\
E\left(\bar{X}^{* 2}(0)\right)=\bar{x}_{0}^{2} \in \mathbb{R}_{+},
\end{array}\right.
\end{aligned}
$$


where,

$$
\begin{aligned}
& \eta(t)=\beta-r-(r+c \beta-k) g(t)-2\left(\Sigma^{\prime} M \lambda\right)^{\prime}\left(\sigma_{Y}^{\prime}-\gamma g(t)\right), \\
& \xi=a-r+\beta-\sigma_{Y}\left(\Sigma^{\prime} M \lambda\right), \pi(t)=\left(\Sigma^{\prime} M \lambda\right)^{\prime}\left(\sigma_{Y}^{\prime}-\gamma g(t)\right) .
\end{aligned}
$$

Note that $E(Y(t))=y_{0} e^{\beta t}$ and $E\left(Y^{2}(t)\right)=y_{0}^{2} e^{\left(2 \beta+\sigma_{Y} \sigma_{Y}^{\prime}\right) t}$. Solving the ODE (45), we find that the expected value of the wealth under optimal control at time $t$ is

$$
\begin{aligned}
& E\left(\bar{X}^{*}(t)\right)= \\
& \bar{x}_{0} e^{(a-r) t}+\frac{(M \lambda)^{\prime} \lambda \omega e^{(a-r) t-r T}\left(e^{(2 r-a) t}-1\right)}{2 r-a} \\
& +c y_{0} \int_{0}^{t} e^{(a-r)(t-s)} \eta(s) e^{\beta s} d s,
\end{aligned}
$$

Solving the ODE (46), we have

$$
\begin{aligned}
& E\left(Y(t) \bar{X}^{*}(t)\right)=x_{0} y_{0} e^{\xi t}+\omega y_{0} \sigma_{Y}\left(\Sigma^{\prime} M \lambda\right) \int_{0}^{t} e^{\xi t-r T+(\beta+r-\xi) s} d s \\
& +c y_{0}^{2} \int_{0}^{t} \sigma_{Y}\left(\sigma_{Y}^{\prime}-\gamma g(s)\right) e^{\xi t+\left(2 \beta+\sigma_{Y} \sigma_{Y}^{\prime}-\xi\right) s} d s,
\end{aligned}
$$

Solving the ODE (47) by using (49), we have

$$
\begin{aligned}
& E\left(\bar{X}^{* 2}(t)\right)=x_{0}^{2} e^{a t}+c x_{0} y_{0} \int_{0}^{t} \eta(s) e^{a t+(\xi-a) s} d s+\int_{0}^{t} G(s) e^{a(t-s)} d s \\
& +c^{2} y_{0}^{2} \int_{0}^{t} \int_{0}^{s} \eta(s) \sigma_{Y}\left(\sigma_{Y}^{\prime}-\gamma g(\tau)\right) e^{a t+(\xi-a) s+\left(2 \beta+\sigma_{Y} \sigma_{Y}^{\prime}-\xi\right) \tau} d \tau d s \\
& +\omega e^{a t}\left(c y_{0} \sigma_{Y}\left(\Sigma^{\prime} M \lambda\right) \int_{0}^{t} \int_{0}^{s} \eta(s) e^{(\xi-a) s+(\beta+r-\xi) \tau-r T} d \tau d s\right. \\
& \left.+2 c y_{0} e^{-r T} \int_{0}^{t} \pi(s) e^{(\beta-a+r) s} d s\right) \\
& +\omega^{2}(M \lambda)^{\prime} \lambda \int_{0}^{t} e^{-2 r(T-s)+a(t-s)} d s,
\end{aligned}
$$

At $t=T$, we have the expected terminal wealth of the PPM to be

$$
\begin{gathered}
E\left(\bar{X}^{*}(T)\right)=\bar{x}_{0} e^{(a-r) T}+\omega\left(1-e^{(a-2 r) T}\right)+ \\
c y_{0} \int_{0}^{T} e^{(a-r)(T-s)} \eta(s) e^{\beta s} d s
\end{gathered}
$$

From (15) and (51) and the definition of $\omega$, we have that $\omega$ is a decreasing function of $\psi$ :

$$
\omega=\frac{e^{-(a-2 r) T}}{2 \psi}+\bar{x}_{0} e^{r T}+c y_{0} e^{r T} \int_{0}^{T} e^{(\beta-a+r) s} \eta(s) d s .
$$


It then follows that

$$
E\left(\bar{X}^{*}(T)\right)=\bar{x}_{0} e^{r T}+c y_{0} e^{r T} \int_{0}^{T} e^{(\beta-a+r) s} \eta(s) d s+\frac{e^{-(a-2 r) T}-1}{2 \psi} .
$$

From (53), we observe that the expected terminal wealth of the PPM is the sum of the wealth that one would get investing the whole portfolio always in both the risky and risk-free asset plus the term, $c y_{0} e^{r T} \int_{0}^{T} e^{(\beta-a+r) s} \eta(s) d s$ that depends both on the goodness of the risky asset, riskless one, initial contribution, and expected growth rate of the contributions of the PPM, plus a term, $\frac{e^{-(a-2 r) T}-1}{2 \psi}$ that depends both on the goodness of the risky asset with respect to the risk-free one and on the weight given to the minimization of the variance. Thus, the higher the expected optimal terminal wealth value, for everything else being equal; the higher the variance minimization parameter, $\psi$, the lower its expected terminal wealth. In the same vain, the higher the growth rate of the contributions of PPM, the higher the terminal wealth of the PPM which is an intuitive result. We therefore conclude that the higher the Sharpe ratio $(M \lambda)^{\prime} \lambda$ and the growth rate of salary of PPM, the higher the terminal wealth of the PPM.

If the entire portfolio should remain in cash account, it implies that $2 r=a$ or $\psi \rightarrow \infty$. In that case, (51) becomes

$$
\begin{aligned}
& \left.E\left(\bar{X}^{*}(T)\right)\right|_{2 r=a}= \\
& \left.E\left(\bar{X}^{*}(T)\right)\right|_{\psi \rightarrow \infty}=\bar{x}_{0} e^{r T}+c y_{0} e^{r T} \int_{0}^{T} e^{(\beta-a+r) s} \eta(s) d s .
\end{aligned}
$$

This shows that $\psi$ only minimize the market price of risk for the PPM.

From (54), we observe that the expected terminal wealth of the PPM is the sum of the wealth that one would get investing the whole portfolio always in the risk-free asset plus the term, $c y_{0} e^{r T} \int_{0}^{T} e^{(\beta-a+r) s} \eta(s) d s$ that depends both on the goodness riskless asset, initial contribution and expected growth rate of the contributions of the PPM.

The optimal portfolio can now be rewritten in-terms of $\psi$ as follows

$$
\begin{aligned}
& \Delta^{\prime *}(t)=-M \lambda\left[\left(\bar{X}^{*}(t)-E\left(\bar{X}^{*}(T)\right) e^{-r(T-t)}+c Y(t) g(t)-\frac{e^{-r(T-t)}}{2 \psi}\right]\right. \\
& -c^{2} Y(t) M \Sigma \sigma_{Y}^{\prime} g(t) .
\end{aligned}
$$

This shows that the portfolio process can be express as a function of the terminal wealth of the PPM and $\psi$. 
(55) is made up of two parts. The first part is the "variational-classical portfolio value" for the PPM. The second part is the intertemporal hedging term that offset any shock to the stochastic salary of the PPM. Interestingly, the portfolio value depend on the expected final total wealth of the PPM and the variance minimizer, $\psi$. At $t=0$, we have

$$
\begin{aligned}
& \Delta^{\prime *}(0)=-M \lambda\left[\left(\bar{x}_{0}-E\left(\bar{X}^{*}(T)\right) e^{-r T}+c y_{0} g(0)-\frac{e^{-r T}}{2 \psi}\right]\right. \\
& -c^{2} y_{0} M \Sigma \sigma_{Y}^{\prime} g(0)
\end{aligned}
$$

\section{Efficient Frontier}

In this section, we derive the efficient frontier for the original mean-variance problem (11). At $t=T$, we have the expected terminal wealth to be

$$
E\left(\bar{X}^{*}(T)\right)=K_{0}(1-\delta) e^{r T}+\omega \delta
$$

where

$$
K_{0}=\bar{x}_{0}+c y_{0} \int_{0}^{T} \eta(s) e^{(\beta-a+r) s} d s, \delta=1-e^{(a-2 r) T} .
$$

The second moment becomes of the PPM's terminal wealth is obtained as

$$
\begin{aligned}
& E\left(\bar{X}^{* 2}(T)\right)=\varphi_{0} e^{a T}+\left(\frac{E\left(\bar{X}^{*}(T)\right)-K_{0}(1-\delta) e^{r T}}{\delta}\right) \varphi_{1} e^{a T} \\
& +\left(\frac{E\left(\bar{X}^{*}(T)\right)-K_{0}(1-\delta) e^{r T}}{\delta}\right)^{2} \delta
\end{aligned}
$$

where,

$$
\begin{aligned}
& \varphi_{0}=\bar{x}_{0}^{2}+c \bar{x}_{0} y_{0} \int_{0}^{T} \eta(s) e^{(\xi-a) s} d s+\int_{0}^{T} E(G(s)) e^{-a s} d s \\
& +c^{2} y_{0}^{2} \int_{0}^{T} \int_{0}^{s} \eta(s) \sigma_{Y}\left(\sigma_{Y}^{\prime}-\gamma g(\tau)\right) e^{(\xi-a) s+\left(2 \beta+\sigma_{Y} \sigma_{Y}^{\prime}-\xi\right) \tau} d \tau d s \\
& \varphi_{1}=c y_{0} \sigma_{Y}\left(\Sigma^{\prime} M \lambda\right) \int_{0}^{T} \int_{0}^{s} \eta(s) e^{(\xi-a) s+(\beta+r-\xi) \tau-r T} d \tau d s \\
& +2 c y_{0} e^{-r T} \int_{0}^{T} \pi(s) e^{(\beta-a+r) s} d s
\end{aligned}
$$

Stat., Optim. Inf. Comput. Vol. 1, December 2013. 
Therefore, the variance can be expressed as a function of the expected optimal final return $E\left(\bar{X}^{*}(T)\right)$ as:

$$
\begin{aligned}
& \operatorname{Var}\left(\bar{X}^{*}(T)\right)=\varphi_{0} e^{a T}+\left(\frac{E\left(\bar{X}^{*}(T)\right)-K_{0}(1-\delta) e^{r T}}{\delta}\right) \varphi_{1} e^{a T} \\
& +\left(\frac{E\left(\bar{X}^{*}(T)\right)-K_{0}(1-\delta) e^{r T}}{\delta}\right)^{2} \delta-\left[E\left(\bar{X}^{*}(T)\right)\right]^{2} \\
& =\varphi_{0} e^{a T}+K_{0}^{2}(1-\delta)^{2} e^{2 r T}+\left(\frac{E\left(\bar{X}^{*}(T)\right)-K_{0}(1-\delta) e^{r T}}{\delta}\right) \varphi_{1} e^{a T} \\
& +\left(\frac{E\left(\bar{X}^{*}(T)\right)-K_{0}(1-\delta) e^{r T}}{\delta}\right)^{2}\left(\delta-\delta^{2}\right) \\
& +2 K_{0}(1-\delta) e^{r T}\left(E\left(\bar{X}^{*}(T)\right)-K_{0}(1-\delta) e^{r T}\right) \\
& =\varphi_{0} e^{a T}+K_{0}^{2}(1-\delta)^{2} e^{2 r T} \\
& +\left(\frac{E\left(\bar{X}^{*}(T)\right)-K_{0}(1-\delta) e^{r T}}{\delta}\right)\left(\varphi_{1} e^{a T}+2 \delta K_{0}(1-\delta) e^{r T}\right) \\
& +\left(\frac{E\left(\bar{X}^{*}(T)\right)-K_{0}(1-\delta) e^{r T}}{\delta}\right)^{2}\left(\delta-\delta^{2}\right) \\
& =\varphi_{0} e^{a T}+K_{0}^{2}(1-\delta)^{2} e^{2 r T}-\frac{\left(\varphi_{1} e^{a T}+2 \delta K_{0} e^{r T}\right)^{2}}{4 \delta} \\
& +\left(\delta-\delta^{2}\right)\left[\frac{\left(\varphi_{1} e^{a T}+2 \delta K_{0}(1-\delta) e^{r T}\right)^{2}}{4\left(\delta-\delta^{2}\right)^{2}}+2\left(\frac{E\left(\bar{X}^{*}(T)\right)-K_{0}(1-\delta) e^{r T}}{\delta}\right)\right. \\
& \left.\left.+\frac{\left(\varphi_{1} e^{a T}+2 \delta K_{0} e^{r T}\right)}{2 \delta}\right)^{2}\right] \\
& +\left(\frac{E\left(\bar{X}^{*}(T)\right)-K_{0}(1-\delta) e^{r T}}{\delta}\right)^{2} \\
& +\left(\delta-\delta^{2}\right)\left[\frac{\left(\varphi_{1} e^{a T}+2 \delta K_{0}(1-\delta) e^{r T}\right)}{4 \delta\left(\bar{X}^{*}(T)\right)-K_{0}(1-\delta) e^{r T}}\right. \\
& +\varphi_{0} e^{a T}+K_{0}^{2}(1-\delta)^{2} e^{2 r T}-\frac{\left(\varphi_{1} e^{a T}+2 \delta K_{0} e^{r T}\right)^{2}}{\delta} \\
& +
\end{aligned}
$$

Therefore, the efficient frontier in mean-variance is obtained as

$$
\begin{aligned}
& E\left(\bar{X}^{*}(T)\right)=2 K_{0} e^{(a-r) T}+\frac{\varphi_{1} e^{a T}}{2\left(1-e^{(a-2 r) T}\right)} \\
& +\frac{\sqrt{\left(1-e^{(a-2 r) T}\right)} \sqrt{\sigma_{\bar{X}^{*}(T)}^{2}-L(T)}}{\sqrt{e^{(a-2 r) T}}}
\end{aligned}
$$

where $L(T)=\varphi_{0} e^{a T}+K_{0}^{2}(1-\delta)^{2} e^{2 r T}-\frac{\left(\varphi_{1} e^{a T}+2 \delta K_{0} e^{r T}\right)^{2}}{4 \delta}$.

Stat., Optim. Inf. Comput. Vol. 1, December 2013. 
(59) is equivalent to

$$
\begin{aligned}
& E\left(\bar{X}^{*}(T)\right)=2 K_{0} e^{\left(r-(M \lambda)^{\prime} \lambda\right) T}+\frac{\varphi_{1} e^{\left(2 r-(M \lambda)^{\prime} \lambda\right) T}}{2\left(1-e^{-(M \lambda)^{\prime} \lambda T}\right)}+ \\
& \sqrt{e^{-(M \lambda)^{\prime} \lambda}-1} \sqrt{\sigma_{\bar{X}^{*}(T)}^{2}-L(T)} .
\end{aligned}
$$

From (62), shows a relation between optimal terminal wealth and its variance. The minimum possible variance, $\sigma_{\bar{X}^{*}(T)}^{2}=L(T) \geq 0$ could be attained when the stakeholder borrows money from the total amount of wealth at time $t=0$ for $T$ years, so that

$$
E\left(\bar{X}^{*}(T)\right)=2 K_{0} e^{\left(r-(M \lambda)^{\prime} \lambda\right) T}+\frac{\varphi_{1} e^{\left(2 r-(M \lambda)^{\prime} \lambda\right) T}}{2\left(1-e^{-(M \lambda)^{\prime} \lambda T}\right)} .
$$

We have that (62) will be a straight line when the initial investment is zero i.e., $x_{0}=0$ which implies that initial contribution is zero as well (i.e., $y_{0}=0$ or $c=0$ ). In that case, the capital market line is

$$
E\left(\bar{X}^{*}(T)\right)=\sqrt{e^{-(M \lambda)^{\prime} \lambda}-1} \sigma_{\bar{X}^{*}(T)} .
$$

The slope, $\sqrt{e^{-(M \lambda)^{\prime} \lambda}-1}$, is the price of risk. (62) shows a linear relationship between the expected optimal terminal wealth and its variance.

Suppose that $x_{0} \neq 0$ and $c=0$, the efficient frontier (62) becomes

$$
E\left(\bar{X}^{*}(T)\right)=2 x_{0} e^{\left(r-(M \lambda)^{\prime} \lambda\right) T}+\sqrt{e^{-(M \lambda)^{\prime} \lambda}-1} \sqrt{\sigma_{\bar{X}^{*}(T)}^{2}-v(T)},
$$

where $v(T)=2 x_{0}^{2} e^{\left(2 r-(M \lambda)^{\prime} \lambda\right) T}+x_{0}^{2} e^{2 r T}\left(e^{2\left(2 r-(M \lambda)^{\prime} \lambda\right) T}-1\right)$.

\section{Numerical Illustration}

In this section, we consider the numerical illustration of our model. The numerical results were obtained by setting $r=0.04, \mu=0.09, \sigma_{B}=0.23, \theta_{B}=0.125, \rho=$ $0.3, \sigma_{S}=0.35, \beta=0.0292, \sigma_{Y_{1}}=0.25, \sigma_{Y_{2}}=0.3, y_{0}=0.9, c=0.075, x_{0}=1$, $\psi=2, T=20$. Figure 1 and Figure 2 show the efficient frontier in mean-variance of the PPM's portfolios for the three classes of assets at different range of values of variance of the terminal wealth. It was observed that the efficient frontier has a parabolic shape as the variance increases, is shown in figure 2. This is due to the present of the stochastic salary of the PPM and to the existence of initial investment. Figure 3, Figure 4 and Figure 5 show the portfolio values of the three classes of assets, inflation-linked bond, stock and cash account, respectively. From the numerical examples, it is observed that more should remain in the riskless asset than the risky ones, inflation-linked bond and stock. 


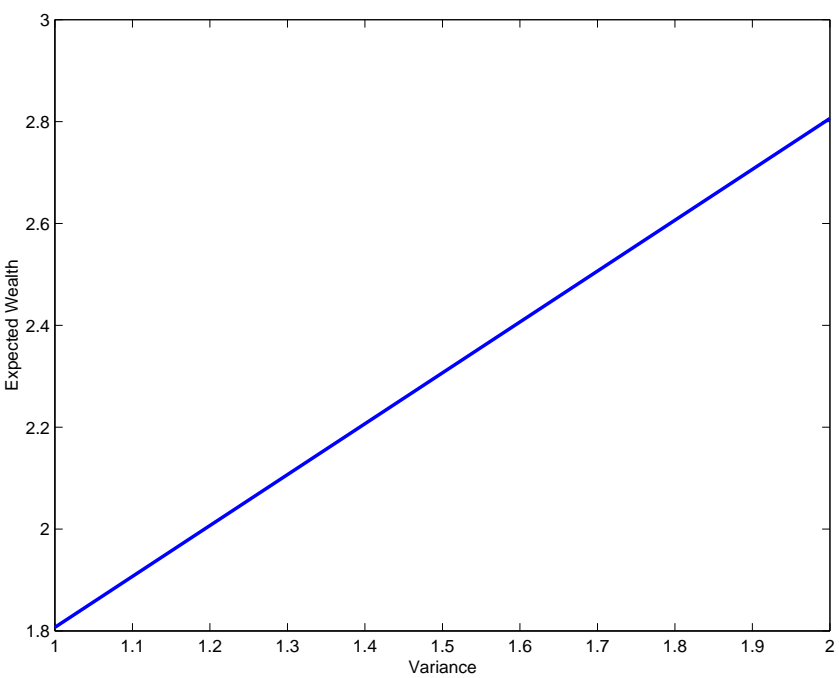

Figure 1. Efficient Frontier.

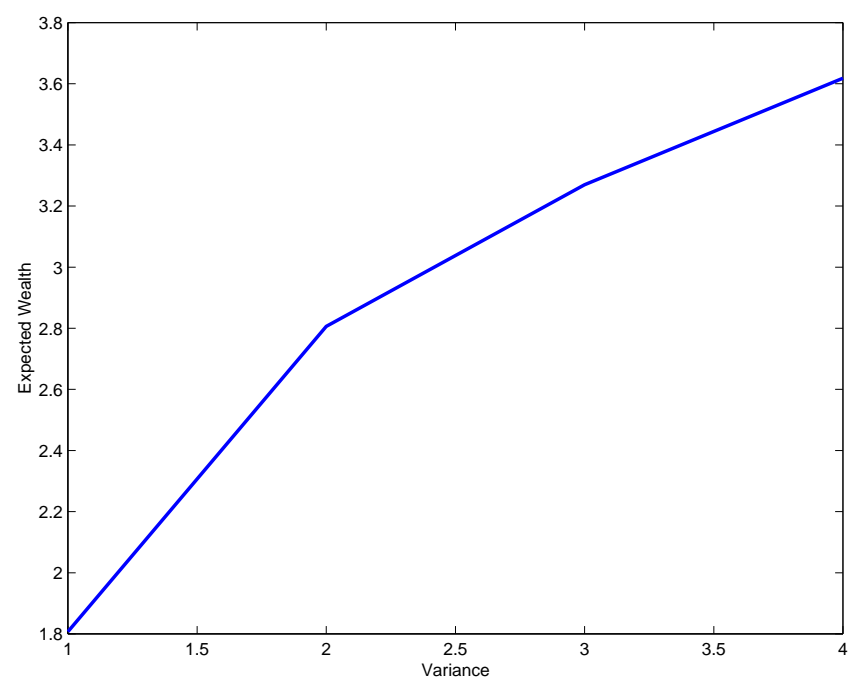

Figure 2. Efficient Frontier, showing clearly the parabolic shape of the frontier.

The total amount of the proportion of initial investment in the risky assets, chosen to maximize the terminal wealth to a certain levels at different values of the variance minimizing parameter, $\psi$, is shown in Table 1 to Table 3 . The investment in the cash account is $x_{0}-\Delta^{* B}(0)-\Delta^{* S}(0)$, that can be obtained 


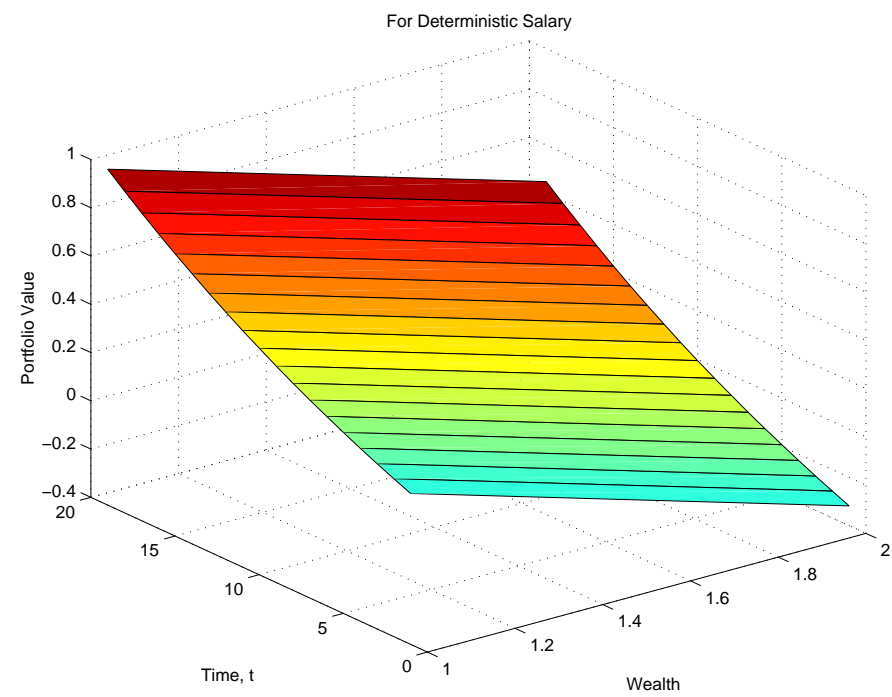

Figure 3. Portfolio Value in inflation-linked bond. We set $\operatorname{Var}\left(\bar{X}^{*}(T)\right)=2$ and $\psi=2$.

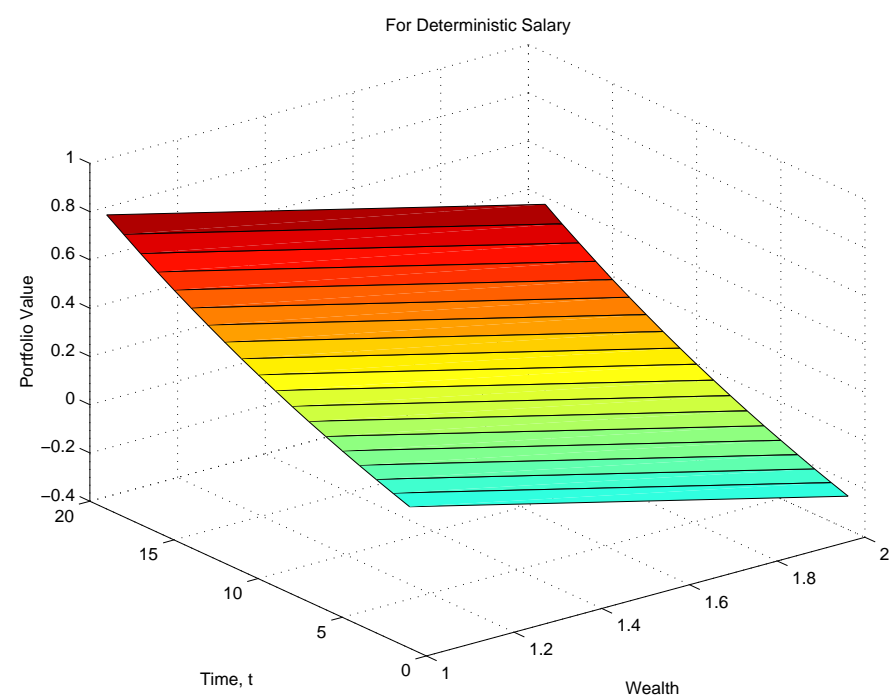

Figure 4. Portfolio Value in Stock. We set $\operatorname{Var}\left(\bar{X}^{*}(T)\right)=2$ and $\psi=2$.

from the tables. There is a case of short-selling, borrowing from cash account to invest in the risky assets is required.

Stat., Optim. Inf. Comput. Vol. 1, December 2013. 


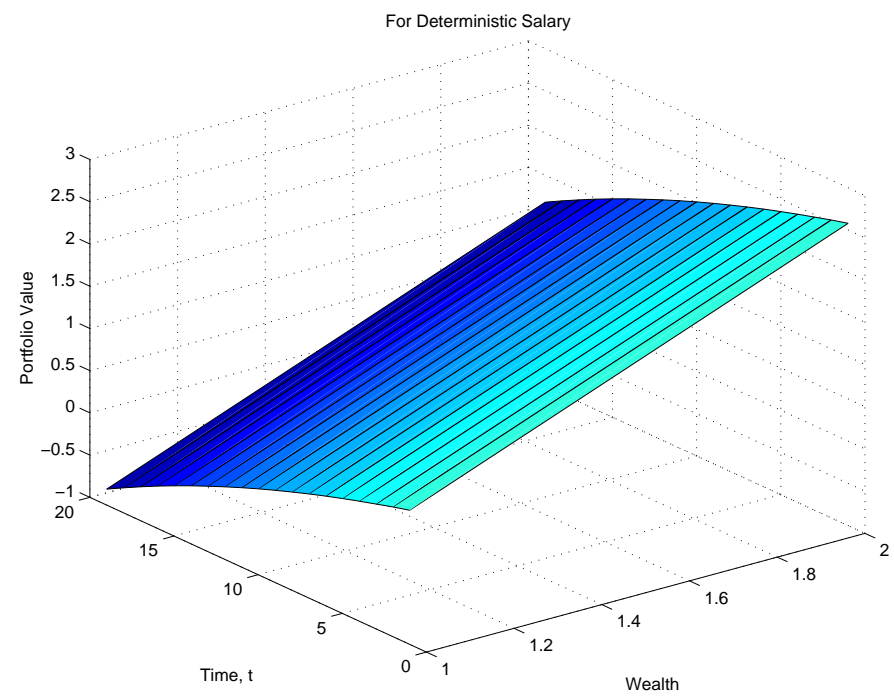

Figure 5. Portfolio Value in Cash Account. We set $\operatorname{Var}\left(\bar{X}^{*}(T)\right)=2$ and $\psi=2$.

Table 1: Initial investment in the risky assets, $\Delta^{* B}(0), \Delta^{* S}(0)$, for $\psi=0.5$

\begin{tabular}{|c|ll|ll|ll|ll|ll|}
\hline \multirow{2}{*}{$E\left(\bar{X}^{*}(T)\right)$} & $\Delta^{* B}$ & $\Delta^{* S}$ & $\Delta^{* B}$ & $\Delta^{* S}$ & $\Delta^{* B}$ & $\Delta^{* S}$ & $\Delta^{* B}$ & $\Delta^{* S}$ & $\Delta^{* B}$ & $\Delta^{* S}$ \\
& $T=1$ & $T=2$ & $T=2$ & $T=5$ & $T=5$ & $T=10$ & $T=10$ & $T=20$ & $T=20$ \\
\hline 0 & 0.0011 & 0.0015 & -0.0194 & -0.0154 & -0.0761 & -0.0622 & -0.1568 & -0.1288 & -0.2769 & -0.2279 \\
1 & 0.5232 & 0.4325 & 0.4823 & 0.3986 & 0.3688 & 0.3050 & 0.2075 & 0.1719 & -0.0327 & -0.0264 \\
2 & 1.0454 & 0.8634 & 0.9840 & 0.8127 & 0.8138 & 0.6722 & 0.5718 & 0.4725 & 0.2115 & 0.1752 \\
3 & 1.5676 & 1.2943 & 1.4857 & 1.2268 & 1.2587 & 1.0395 & 0.9361 & 0.7732 & 0.4557 & 0.3767 \\
4 & 2.0897 & 1.7253 & 1.9874 & 1.6408 & 1.7037 & 1.4067 & 1.3004 & 1.0739 & 0.6999 & 0.5783 \\
5 & 2.6119 & 2.1562 & 2.4891 & 2.0548 & 2.1487 & 1.7739 & 1.6647 & 1.3745 & 0.9441 & 0.7798 \\
\hline
\end{tabular}

Table 2: Initial investment in the risky assets, $\Delta^{* B}(0), \Delta^{* S}(0)$, for $\psi=1$

\begin{tabular}{|c|cc|cc|cc|cc|cc|}
\hline$E\left(\bar{X}^{*}(T)\right)$ & $\Delta^{* B}$ & $\Delta^{* S}$ & $\Delta^{* B}$ & $\Delta^{* S}$ & $\Delta^{* B}$ & $\Delta^{* S}$ & $\Delta^{* B}$ & $\Delta^{* S}$ & $\Delta^{* B}$ & $\Delta^{* S}$ \\
\hline 0 & -0.2600 & -0.2140 & -0.2703 & -0.2224 & -0.2986 & -0.2458 & -0.3390 & -0.2791 & -0.3990 & -0.3287 \\
1 & 0.2621 & 0.2170 & 0.2314 & 0.1916 & 0.1463 & 0.1214 & 0.0253 & 0.0216 & -0.1548 & -0.1271 \\
2 & 0.7843 & 0.6479 & 0.7331 & 0.6057 & 0.5913 & 0.4886 & 0.3896 & 0.3222 & 0.0894 & 0.0744 \\
3 & 1.3065 & 1.0789 & 1.2348 & 1.0197 & 1.0363 & 0.8559 & 0.7540 & 0.6229 & 0.3336 & 0.2759 \\
4 & 1.8287 & 1.5098 & 1.7365 & 1.4338 & 1.4812 & 1.2231 & 1.1183 & 0.9235 & 0.5778 & 0.4775 \\
5 & 2.3508 & 1.9408 & 2.2382 & 1.8478 & 1.9262 & 1.5903 & 1.4826 & 1.2242 & 0.8220 & 0.6790 \\
\hline
\end{tabular}

Table 3: Initial investment in the risky assets, $\Delta^{* B}(0), \Delta^{* S}(0)$, for $\psi=10$

\begin{tabular}{|c|cc|cc|cc|cc|cc|}
\hline$E\left(\bar{X}^{*}(T)\right)$ & $\Delta^{* B}$ & $\Delta^{* S}$ & $\Delta^{* B}$ & $\Delta^{* S}$ & $\Delta^{* B}$ & $\Delta^{* S}$ & $\Delta^{* B}$ & $\Delta^{* S}$ & $\Delta^{* B}$ & $\Delta^{* S}$ \\
& $T=1$ & $T=2$ & $T=2$ & $T=5$ & $T=5$ & $T=10$ & $T=10$ & $T=20$ & $T=20$ \\
\hline 0 & -0.4950 & -0.4079 & -0.4960 & -0.4087 & -0.4989 & -0.4111 & -0.5029 & -0.4144 & -0.5009 & -0.4194 \\
1 & 0.0272 & 0.0231 & 0.0057 & 0.0053 & -0.0539 & -0.0438 & -0.1386 & -0.1137 & -0.2647 & -0.2178 \\
2 & 0.5493 & 0.4540 & 0.5074 & 0.4194 & 0.3911 & 0.3234 & 0.2257 & 0.1869 & -0.0205 & -0.0163 \\
3 & 1.0715 & 0.8849 & 1.0091 & 0.8334 & 0.8360 & 0.6906 & 0.5900 & 0.4876 & 0.2237 & 0.1853 \\
4 & 1.5937 & 1.3159 & 1.5108 & 1.2475 & 1.2810 & 1.0578 & 0.9543 & 0.7882 & 0.4679 & 0.3868 \\
5 & 2.1158 & 1.7468 & 2.0124 & 1.6615 & 1.7260 & 1.4251 & 1.3186 & 1.0889 & 0.7121 & 0.5883 \\
\hline
\end{tabular}

Stat., Optim. Inf. Comput. Vol. 1, December 2013. 


\section{Conclusion}

We have analyzed the management of a pension funding process of a defined contributory pension scheme where the salary of the PPM is stochastic. The objective is to determine investments strategies maximizing the expected terminal wealth and at the same time minimizing the variance of the terminal wealth. The problem is formulated as a mean-variance optimization problem and was solved by means of dynamic programming principles.

The portfolio is made up of two parts. The first part is the "variational-classical portfolio value" for the PPM. The second part is the intertemporal hedging term that offset any shock to the stochastic salary of the PPM. Interestingly, the portfolio value was found to depend on the expected final total wealth of the PPM and the variance minimizing parameter.

We found that the PPM's capital line can be attained when the initial capital and initial contributions are zeros. The efficient frontier was found to be a parabolic in shape, due to the present of initial capital and the existence of stochastic salary of the PPM.

A numerical illustration of the analytical results and models are established in this paper.

\section{Acknowledgement}

I acknowledge the interesting comments and suggestions of the anonymous referees which contributed tremendously to the improvement of this paper.

\section{REFERENCES}

1. I. Bajeux-Besnainou and R. Portait, Dynamic asset allocation in a mean-variance framework, Management Science, 44(1998), S79-S95.

2. P. Battocchio and F. Menoncin, Optimal pension management in a stochastic framework, Insurance: Mathematics and Economics, 34(2004), pp. 79-95.

3. T. Bielecky, H. Jim, S. Pliska, and X. Zhou, Continuous-time mean-variance portfolio selection with bankruptcy prohibition, Mathematical Finance, 15(2005), pp. 213-244.

4. D. Blake, D. Wright and Y. Zhang, Optimal funding and investment strategies in defined contribution pension plans under Epstein-Zin utility, Discussion paper, The pensions Institute, Cass Business School, City University, UK (2008).

5. J. F. Boulier, S. J. Huang and G. Taillard, Optimal management under stochastic interest rates: The case of a protected defined contribution pension fund, Insurance: Mathematics and Economics, 28(2001), pp. 173-189.

6. A. J. G. Cairns, D. Blake, and K. Dowd, Stochastic lifestyling: Optimal dynamic asset allocation for defined contribution pension plans. Journal of Economic Dynamic and Control, 30(2006), pp. 843-377.

7. M. Chiu and D. Li, Asset and liability management under a continuous-time mean-variance optimization framework, Insurance: Mathematics and Economics, 39(2006), pp. 330-355. 
8. G. Deelstra, M. Grasselli and P. Koehl, Optimal investment strategies in a CIR framework. Journal of Applied Probability, 37(2000), pp. 936-946.

9. P. Devolder, M. Bosch Princep and I. D. Fabian, Stochastic optimal control of annuity contracts, Insurance: Mathematics and Economics, 33(2003), pp. 227-238.

10. M. Di Giacinto, S. Federico and F. Gozzi, Pension funds with a minimum guarantee: $a$ stochastic control approach, Finance and Stochastic, (2010).

11. J. Gao, Stochastic optimal control of DC pension funds, Insurance: Mathematics and Economics, 42(2008), pp. 1159-1164.

12. R. Gerrard, S. Haberman and E. Vigna, Optimal investment choices post retirement in a defined contribution pension scheme, Insurance: Mathematics and Economics, 35(2004), pp. 321-342.

13. S. Haberman and E. Vigna, Optimal investment strategies and risk measures in defined contribution pension schemes, Insurance: Mathematics and Economics, 31(2002), pp. 35-69.

14. B. H $\phi$ jgaard and E. Vigna, Mean-variance portfolio selection and efficient frontier for defined contribution pension schemes, Technical report R-2007-13, Department of Mathematical Sciences, Aalborg University, 2007.

15. R. Josa-Fombellida and J. Rincón-Zapatero, Mean-variance portfolio and contribution selection in stochastic pension funding, European Journal of Operational Research, 187(2008), pp. 120-137.

16. R. Korn and M. Krekel, Optimal portfolios with fixed consumption or income streams. Working paper, University of Kaiserslautern, 2001.

17. D. Li and W.-L. Ng, Optimal dynamic portfolio selection: multiperiod mean-variance formulation, Mathematical Finance, 10(2000), pp. 387-406.

18. H. Markowitz, Portfolio selection, Journal of finance, 7(1952), pp. 77-91.

19. H. Markowitz, Portfolio selection: efficient diversification of investments, New York, Wiley, 1959.

20. C. I. Nkeki, On optimal portfolio management of the accumulation phase of a defined contributory pension scheme. Ph.D thesis, Department of Mathematics, University of Ibadan, Ibadan, Nigeria, 2011.

21. C. I. Nkeki and C. R. Nwozo, Variational form of classical portfolio strategy and expected wealth for a defined contributory pension scheme. Journal of Mathematical Finance 2(2012), 132-139.

22. H. Richardson, A minimum variance result in continuous trading portfolio optimization, Management Science, 35(1989), pp. 1045-1055.

23. E. Vigna, On efficiency of mean-variance based portfolio selection in DC pension schemes, Collegio Carlo Alberto Notebook 154, 2010.

24. X. Zhou and D. Li, Continuous-time mean-variance portfolio selection: A stochastic LQ framework, Applied Mathematics and Optimization, 42(2000), pp. 19-33.

25. Nigerian Pension Reform Act, 2004. 